\title{
A Therapeutic Equivalence of Two Formulations of the Fixed Combination of Benzydamine Hydrochloride and Cetylpyridinium Chloride in the Treatment of Sore Throat Associated with Upper Respiratory Tract Infection
}

\author{
Marko Boh ${ }^{1}$, Ivan Gordeev ${ }^{2}$ and Mateja Bulc ${ }^{3 *}$ \\ ${ }^{1}$ Krka, d. d., Novo mesto, Novo mesto, Slovenia \\ ${ }^{2}$ Federal State Budgetary Institution The Russian National Research Medical University n.a. N.I. Piragov, Moscow, Russian Federation \\ ${ }^{3}$ Communitiy Health Centre Ljubljana-Šiška, Ljubljana, Slovenia
}

${ }^{*}$ Corresponding author: Marko Boha, Krka, d. d., Novo mesto, Novo mesto, Slovenia

Received: March 09, 2021; Accepted: March 17, 2021; Published: March 22, 2021

\begin{abstract}
Background: The combination of Cetylpyridinium chloride and Benzydamine hydrochloride (CPC/BH) has antiseptic, antimicrobial, anti-inflammatory and analgesic properties. We have investigated whether the fixed combination in the form of lozenges is therapeutically equivalent to the orosoluble tablet formulation in a randomized, parallel, partially double-blind, three-arm, placebo controlled clinical trial in patients with sore throat due to upper respiratory tract infection.
\end{abstract}

Methods: Clinical assessment was made within 3 hours after the initial single dose drug administration. Thereafter, subjects underwent the therapy according to the prescribed schedule for additional 4-7 days, primarily to assess the safety profile of CPC/BH. Multiple measurements using Visual Analogue Scale (VAS) were performed to assess sore throat pain intensity, while categorical Sore Throat Pain Relief Scale (STPRS) was used to assess sore throat pain relief within three hours after the initial dose administration. Tonsillopharyngitis Assessment Score was used to assess the resolution of upper respiratory tract infection after 4 and 7 days of therapy.

Results: Altogether 291 patients aged 39,6 $\pm 11,5$ years with sore throat were randomly assigned to receive CPC/BH lozenges (118 patients), CPC/BH orosoluble tablets (116 patients) or placebo (57 patients). Both formulations were significantly more effective than placebo in reducing sore throat pain intensity at 1,2 and 3 hours after the initial dose administration. The two formulations were found to be equivalent in terms of therapeutic efficacy at all three measurement points. Similarly, Total Pain Relief over the time interval of 15 minutes to 3 hours after the initial dose was greater in both formulations in comparison with placebo and no significant difference between the two formulations. Altogether $28,1 \%$ of patients in placebo group, 59,3\% in CPC/BH lozenges group and $47,4 \%$ in $\mathrm{CPC} / \mathrm{BH}$ orosoluble tablets group were considered therapy responders. Again, both treatments were significantly better than placebo with no significant difference between the two formulations. The mean time to onset of sore throat pain relief was significantly shorter in both formulations with comparison to placebo. At day 5 of treatment, the disease was completely resolved in $31,6 \%$ of subjects in the placebo group and in $53,4 \%$ patients treated with both $\mathrm{CPC} / \mathrm{BH}$ formulations ( $\mathrm{p}=0,007)$. At day 8 , the disease was resolved in $66,7 \%, 89,0 \%$ and $81,0 \%$ of patients in placebo, $\mathrm{CPC} / \mathrm{BH}$ lozenges and $\mathrm{CPC} / \mathrm{BH}$ orosoluble tablets groups, respectively. There was similar statistical significance between the groups as at day 5 . Altogether, 10 therapy-related adverse events have been reported. There were all of mild intensity, none of them was serious and they were relatively evenly distributed between the treatment groups.

Conclusions: The therapeutic equivalence between the lozenges and orosoluble tablets formulation of CPC/BH was demonstrated in terms of sore throat pain intensity reduction, sore throat pain relief, percent of responders and time to the pain relief onset after the initial dose and in terms of the disease resolution after 4 and 7 days of treatment. Both formulations were superior to placebo. Both CPC/BH formulations have similar safety profile to placebo.

Keywords: Sore throat, Upper respiratory tract infection, Cetylpyridinium chloride, Benzydamine hydrochloride, Analgesic, Anti-inflammatory, Therapeutic equivalence

\section{Introduction}

Upper respiratory tract infections are one of the most common acute infectious diseases including acute pharyngitis. The latter is one of the commonest complaints at the general practitioner level of medical care, even so, most of the people with pharyngitis do not seek medical help [1,2]. Predominantly, pharyngitis or tonsillopharyngitis is caused by viral infection. In less than $20 \%$ of cases bacteria are involved [3]. Hence, the use of antibiotics for the treatment of acute pharyngitis is questionable and in many cases inappropriate $[4,5]$. 
Cetylpyridinium chloride (CPC) belongs to a family of quaternary ammonium bases. It exerts its antimicrobial effect through nonspecific interaction at the bacterial cytoplasmic membrane [6]. CPC is a broad-spectrum antiseptic that has both a bactericidal and virucidal effects. In addition to its antiseptic effect, it also has emulsifying and detergent properties and helps to lubricate and soothe the painful area. It penetrates the least accessible places on the mucous membrane. This is an important factor in the treatment of mouth and throat infections [7]. Benzydamine hydrochloride (BH), a nonsteroid anti-inflammatory drug (NSAID) has analgesic, anaesthetic, anti-inflammatory and antimicrobial properties. It is a weak inhibitor of the synthesis of prostaglandins but it has several properties, which may contribute to its anti-inflammatory activity [8]. Clinical studies with benzydamine used for management of inflammatory oropharyngeal conditions have demonstrated results, mainly exerted as a more rapid resolution of pain and dysphagia and reduction of inflammation $[9,10]$.

Krka, d. d., Novo mesto has developed the fixed dose combination of $3 \mathrm{mg} \mathrm{CPC}$ and $1 \mathrm{mg} \mathrm{BH}$ in the form of lozenges. The therapeutic regimen is one lozenge four times daily, i.e. each 3 hours. The principal aim of our study was to investigate whether the $\mathrm{CPC} / \mathrm{BH}$ lozenges are therapeutically equivalent to the reference marketed fixed combination dose of $3 \mathrm{mg} \mathrm{CPC}$ and $1 \mathrm{mg} \mathrm{BH}$ in the form of orosoluble tablets, which has the identical dose regimen, in patients with sore throat due to upper respiratory tract infection. The second aim was to demonstrate the superior effect of both $\mathrm{CPC} / \mathrm{BH}$ formulations over placebo.

\section{Patients and Methods}

This was a randomized, parallel, partially double blind, three arm, placebo-controlled, equivalence, multicenter, international trial conducted at 11 clinical sites in Russian Federation and Slovenia. Due to issues with the dissolution time, the blind was only assured for the lozenges (i.e. placebo was in the form of lozenges). Even so, orosoluble tablets were not on the market in participating countries at the time of the study conduct so the patients could not have recognized the reference product.

In order to be enrolled, male or female patients, aged 18 to 65 years, should have had the onset of moderate to severe sore throat six days or less before the screening/enrolment visit. They had signs and symptoms of acute tonsillopharyngitis. They have signed written informed consent to participate in the trial. Among the noninclusion criteria were streptococcal tonsillitis assessed with rapid antigen detection test in patients with severe pathology of changes in pharyngeal region, increased body temperature that needed antipyretic treatment (more than 38, 5 degrees Celsius), oropharyngeal lesions such as tumours, purulent necrotic process or aphtous ulcers, any evidence of mouth breathing or coughing which could compromise respiratory function and worsen sore throat and concomitant therapy that could bias the assessment of therapeutic efficacy and safety.

Patients have undergone two or three visits at the outpatient clinics. At the first visit, the initial single dose of study therapy has been administered and measurements of pain intensity and pain relief performed over three hours. Thereafter, patients continued with the usual therapeutic regimen for 4 or 7 days. There were two additional visits on day 5 and 8 of the study to assess safety and efficacy of the treatments. Patient whose condition has resolved after 4 days of therapy, concluded the therapy on day 5 , while those with persisting signs and symptoms of the disease have been treated for another three days.

The key parameter of the assessment of efficacy endpoints was Sore Throat Pain Intensity (STPI). It was assessed by Visual Analog Scale (VAS) that ranges from zero (not sore) to 100 (very sore) in millimetres [11]. The measurement points (in minutes) at the initial visit were zero (baseline at the time of the therapy intake), 15, 30, 45, $60,75,90,105,120,135,150,165$, and 180 .

Primary efficacy endpoint was a difference between the STPI at one hour after the administration of the initial dose and baseline STPI value while secondary endpoints included the difference in STPI between STPI at two hours and three hours, and baseline STPI value. Another secondary endpoint was percent of responders defined by the threshold STPI reduction at first, second and third hour after the therapy administration.

At each of the two following visits, a single STPI measurement has been made to yield the combined tertiary efficacy endpoint of percent of patients with the disease resolution.

Another parameter of efficacy assessment was sore throat pain relief (STPAR) measured at the initial visit by the categorical Sore Throat Pain Relief Scale (STPARS) containing seven categories of pain relief description [12]. The measurement points with STPARS were identical to VAS except the baseline measurement where no relief could has been assessed hence no assessment with STPARS was made. A secondary efficacy endpoint Total pain relief (TOTPAR) was defined as the area under the curve (AUC) in the time interval from 15 minutes to 180 minutes after the initial dose administration. At each of the two following visits, a single STPRS measurement has been made to yield the combined tertiary efficacy endpoint of percent of patients with the disease resolution.

Tonsillopharyngitis assessment score (TPAS) [11,13] has been assessed to evaluate objective signs of the condition, which served as the assessment for the inclusion criterion and for two tertiary efficacy endpoints. It consists of five features assessed by the investigator: body temperature, oropharyngeal colour, oropharyngeal enanthemas, cervical adenopathy and cervical adenitis. To assess the safety profile, an interview and physical inspection were used.

Statistically, this study has been based on equivalence design. The null hypothesis for the primary efficacy endpoint is non-equivalence between the two $\mathrm{CPC} / \mathrm{BH}$ formulations, and the alternative hypothesis is equivalence defined by pre-defined equivalence margin $(\delta)$ of 13 millimetres of VAS score [14]. Assessment of the hypotheses was based on a two-sided $95 \%$ confidence interval for the treatment difference. It was to be concluded that the two formulations are equivalent if the lower and upper bounds of the $95 \%$ confidence interval for the treatment difference lie entirely within $(-\delta, \delta)$ interval. In order to assure the assay sensitivity as an inherent part of the equivalence 
design, a superiority alternative hypothesis of both formulations against placebo was tested. In order to achieve the study power of $80 \%$ and type I error $(\alpha<0.025)$, minimally 273 subjects should have finished the primary point assessment. Due to expected minimal effect of placebo, a balanced treatment allocation of 1:2:2 was used for placebo, and each of the formulations, respectively.

\section{Results}

Altogether 291 patients were enrolled and underwent the single dose efficacy assessment. The baseline characteristics are displayed in the Table 1 .

Out of 291 patients, 143 successfully completed the treatment at Visit 2 with no major protocol violation, while 143 concluded the treatment at visit 3 . There were 5 drop-outs.

The mean difference between the two formulations in STPI at 1 hour (primary efficacy endpoint) has been $3,38 \mathrm{~mm}$ in favour of $\mathrm{CPC} / \mathrm{BH}$ lozenges. The corresponding 95\% CI were - 1,73 and 8,49 $\mathrm{mm}$ and were therefore entirely within the equivalence margin. The mean difference between $\mathrm{CPC} / \mathrm{BH}$ orosoluble tablets and placebo has been $19,74 \mathrm{~mm}$. The difference was statistically significant $(\mathrm{p}<0,001)$. Likewise, the mean difference between $\mathrm{CPC} / \mathrm{BH}$ lozenges and placebo was statistically significant. It has been $23,11 \mathrm{~mm}(\mathrm{p}<0,001)$. The two secondary efficacy endpoints-STPI difference at 2 hours and STPI difference at 3 hours have shown similar results and therefore

Table 1: Baseline characteristics.

\begin{tabular}{|c|c|c|c|c|}
\hline & $\begin{array}{c}\text { Placebo } \\
\mathrm{N}=\mathbf{5 7}\end{array}$ & $\begin{array}{c}\mathrm{CPC} / \mathrm{BH} \\
\text { Orosoluble tablets } \\
\mathrm{N}=116\end{array}$ & $\begin{array}{c}\mathrm{CPC} / \mathrm{BH} \\
\text { Lozenges } \\
\mathrm{N}=118\end{array}$ & $\begin{array}{c}\text { Total } \\
\mathrm{N}=291\end{array}$ \\
\hline $\begin{array}{l}\text { Age (years) } \\
\text { Mean (SD) } \\
\text { Min/max }\end{array}$ & $\begin{array}{c}39,9(10,80) \\
24 / 62\end{array}$ & $\begin{array}{c}40,5(12,23) \\
19 / 65\end{array}$ & $\begin{array}{c}38,5(11,07) \\
19 / 64\end{array}$ & $\begin{array}{c}39,55(11,49) \\
19 / 65\end{array}$ \\
\hline $\begin{array}{l}\text { Gender (\%) } \\
\text { Male } \\
\text { Female }\end{array}$ & $\begin{array}{l}35 \\
65\end{array}$ & $\begin{array}{l}36 \\
64\end{array}$ & $\begin{array}{l}26 \\
74\end{array}$ & $\begin{array}{l}32 \\
68\end{array}$ \\
\hline $\begin{array}{l}\text { Body temperature }\left({ }^{\circ} \mathrm{C}\right) \\
\text { mean }(\mathrm{SD}) \\
\min / \max \end{array}$ & $\begin{array}{c}37,49(0,3) \\
36,7 / 38,3\end{array}$ & $\begin{array}{c}37,49(0,4) \\
36,6 / 38,4\end{array}$ & $\begin{array}{c}37,49(0,4) \\
36,4 / 38,4\end{array}$ & $\begin{array}{c}37,49(0,4) \\
36,4 / 38,4\end{array}$ \\
\hline $\begin{array}{l}\text { TPA score-baseline } \\
\text { mean }(\mathrm{SD}) \\
\text { median } \\
\mathrm{min} / \max \end{array}$ & $\begin{array}{c}5,1(0,9) \\
5,0 \\
4 / 8\end{array}$ & $\begin{array}{c}5,1(1,1) \\
5,0 \\
4 / 9\end{array}$ & $\begin{array}{c}5,2(0,9) \\
5,0 \\
4 / 7\end{array}$ & $\begin{array}{l}- \\
- \\
-\end{array}$ \\
\hline $\begin{array}{l}\text { STPI }(\mathrm{mm}) \\
\text { mean }(\mathrm{SD}) \\
\min / \max \end{array}$ & $\begin{array}{c}80,0(10,28) \\
64 / 100\end{array}$ & $\begin{array}{c}78,2(10,02) \\
52 / 100\end{array}$ & $\begin{array}{c}77,6(9,89) \\
60 / 100\end{array}$ & $\begin{array}{c}78,3(10,02) \\
52 / 100\end{array}$ \\
\hline
\end{tabular}

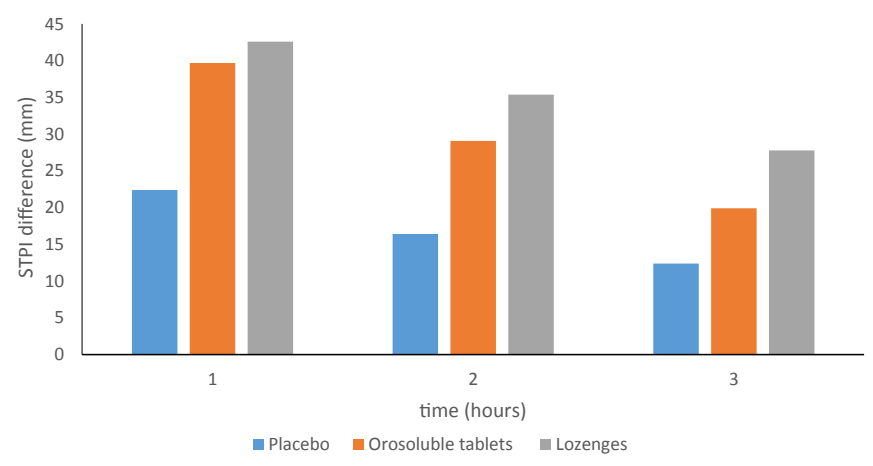

Figure 1: STPI difference at hour 1,2 and 3. corroborated the outcome with the primary efficacy endpoint. The primary and secondary efficacy endpoints denoting STPI difference at hours 1, 2 and 3 are shown in the Figure 1.

The STPAR score values over the time interval of 15-180 minutes, which represent the basis for the TOTPAR calculation, are shown in the Figure 2. The TOTPAR over the corresponding time interval has been significantly higher in the therapeutic groups who were administered the two $\mathrm{CPC} / \mathrm{BH}$ formulations in comparison with placebo group. There were no significant differences between the two formulations.

Altogether, 59.2 percent of patients who have taken $\mathrm{CPC} / \mathrm{BH}$ lozenges and 47.7 percent of those who were treated with $\mathrm{CPC} / \mathrm{BH}$ orosoluble tablets have responded adequately to the therapy. There were no significant difference between the two therapeutic groups, whereas both formulations were significantly better that placebo (Figure 3).

After 4 days of treatment, sore throat and signs of tonsillopharyngitis have resolved in $31,6 \%$ of patients in placebo group, and in both CPC/ $\mathrm{BH}$ formulation groups in $53,4 \%$ of subjects. The difference between placebo and active treatments was significant $(\mathrm{p}=0.007)$. After 7 days of treatment, the disease was resolved in $66,7 \%, 89,0 \%$ and $81,0 \%$ of patients in placebo, $\mathrm{CPC} / \mathrm{BH}$ lozenges and $\mathrm{CPC} / \mathrm{BH}$ orosoluble tablets groups, respectively. There was similar statistical significance between the groups as after 4 days of therapy.

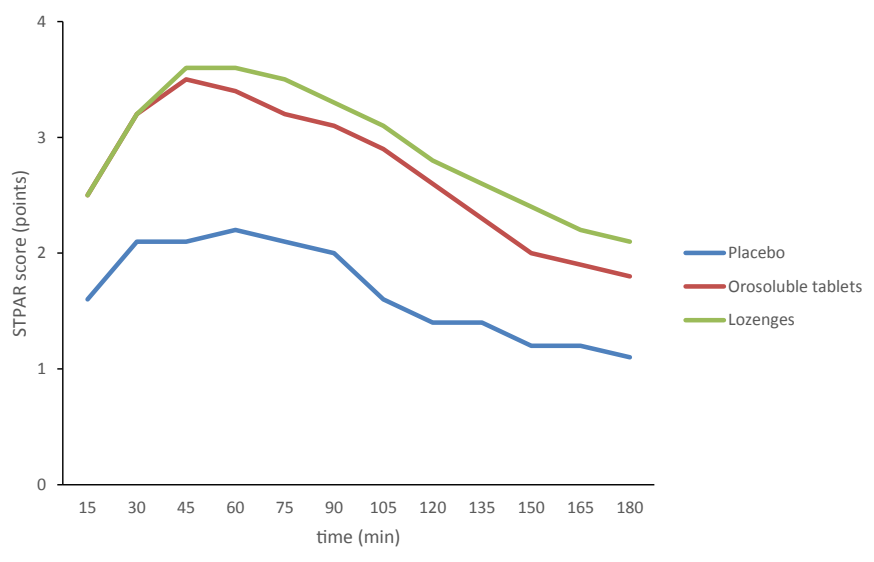

Figure 2: TSPAR score over the time interval of 15-180 minutes.

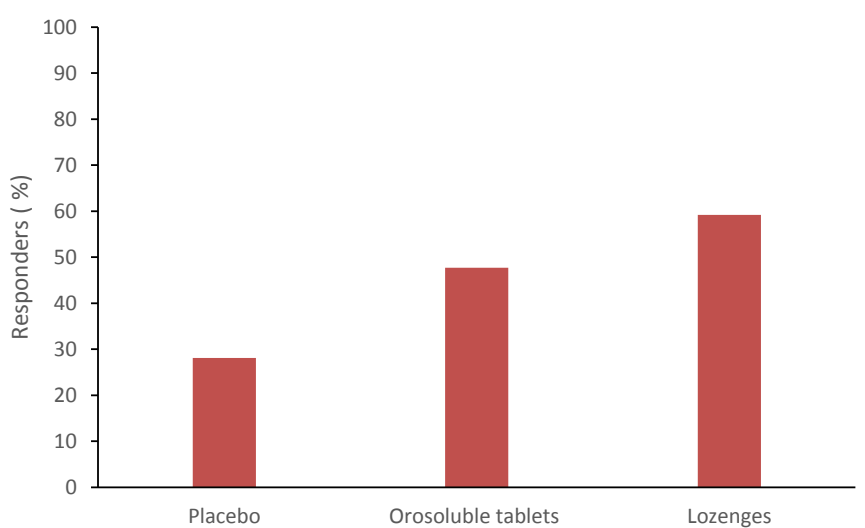

Figure 3: Percent of responders after the initial dose therapy administration. 
Marko Boh (2021) A Therapeutic Equivalence of Two Formulations of the Fixed Combination of Benzydamine Hydrochloride and Cetylpyridinium Chloride in the Treatment of Sore Throat Associated with Upper Respiratory Tract Infection

\begin{tabular}{|c|c|c|c|c|c|c|}
\hline & & & & Treatment & & \\
\hline & & & $\begin{array}{c}\begin{array}{c}\text { Placebo } \\
\mathrm{N}=57\end{array}\end{array}$ & $\begin{array}{c}\begin{array}{c}\text { Orosoluble } \\
\text { tablets }\end{array} \\
\mathrm{N}=116\end{array}$ & $\begin{array}{c}\text { Lozenges } \\
\mathrm{N}=118\end{array}$ & $\begin{array}{c}\text { Total } \\
\mathrm{N}=291\end{array}$ \\
\hline \multirow{9}{*}{ Gastrointestinal disorders } & & Diarrhoea & 0 & $1(0,9 \%)$ & 0 & $1(0,3 \%)$ \\
\hline & & Dry mouth & 0 & 0 & $2(1,7 \%)$ & $2(0,7 \%)$ \\
\hline & & Heartburn & 0 & 0 & $1(0,9 \%)$ & $1(0,3 \%)$ \\
\hline & & Meteorism & $1(1,8 \%)$ & 0 & 0 & $1(0,3 \%)$ \\
\hline & & Nausea & 0 & $1(0,9 \%)$ & 0 & $1(0,3 \%)$ \\
\hline & & Numbness of tongue & 0 & $1(0,9 \%)$ & 0 & $1(0,3 \%)$ \\
\hline & & Parageusia & 0 & $1(0,9 \%)$ & 0 & $1(0,3 \%)$ \\
\hline & & Stomach pain & 0 & $1(0,9 \%)$ & 0 & $1(0,3 \%)$ \\
\hline & \multicolumn{2}{|l|}{ Total } & $1(1,8 \%)$ & $5(4,3 \%)$ & $3(2,5 \%)$ & $9(3,1 \%)$ \\
\hline \multirow{2}{*}{ Skin and subcutaneous tissue disorders } & & Erythema facial & $1(1,8 \%)$ & 0 & 0 & $1(0,3 \%)$ \\
\hline & \multicolumn{2}{|l|}{ Total } & $1(1,8 \%)$ & 0 & 0 & $1(0,3 \%)$ \\
\hline
\end{tabular}

There were altogether 10 drug-related adverse events reported. The overall incidence per treatment group was 3,51\%, 4,31\% and $2,54 \%$ for placebo, $\mathrm{CPC} / \mathrm{BH}$ orosoluble tablets and $\mathrm{CPC} / \mathrm{BH}$ lozenges, respectively. Due to a low number of drug-related adverse events, a comparative analysis was not performed.

The summary of drug-related adverse events is displayed in the Table 2. There were no patients who would be unable to finish the assessment due to clinically significant adverse events. There were no deaths or any other serious adverse events in this study.

\section{Discussion and Conclusions}

In this clinical trial, two formulations containing fixed combination of CPC $1 \mathrm{mg}$ and $\mathrm{BH} 3 \mathrm{mg}$ have been tested to establish the therapeutic equivalence of the two formulations with respect to efficacy and safety profile. The Orosoluble tablets have already been on the EU market at the time of the trial conduct contrary to lozenges pharmaceutical form of lozenges that was developed by Krka, d. d. Clinical data on the fixed combination have been rather scarce [15] including lack of placebo controlled studies. In order to assure essay sensitivity to established efficacy of the marketed pharmaceutical form, we had to include placebo arm to demonstrate the superiority of the reference drug [16].

As for the study methodology, all the key parameters of efficacy assessment including Sore throat pain intensity and Sore throat pain relief scales and Tonsillopharyngitis assessment score are wellestablished methods to assess efficacy in the setting of the claimed indication. They are corroborated with the research which has established their value as an assessment $[11,17,18]$ as well as with studies of different medicinal products with similar indication profile $[12,19]$.

The principal and decisive endpoint in the efficacy equivalence assessment in this trial was a single one. It represents the difference in pain intensity at the pre-determined time point as recommended by the EU Note on guidance on clinical trials of nociceptive pain.
The results of the comparison analysis, which included alternative hypothesis of equivalence, has demonstrated unequivocally that the two formulations are equivalent. The assay sensitivity has been assured by a clear-cut superiority of both active treatments over placebo in the primary efficacy endpoint analysis. The superiority of both $\mathrm{CPC} / \mathrm{BH}$ formulations over the placebo in the pain intensity reduction persisted also after 2 and 3 hours as assessed by secondary efficacy endpoints. The secondary endpoint TOTPAR is an indicator of the pain control throughout the entire dosing interval. It is one of the main endpoints of sore throat pain model for the assessment of sore throat pain, which is commonly used in the comparative therapeutic intervention trials $[17,20]$. The percent of patients that respond adequately to the therapy is a very relevant endpoint in the trials that investigate the comparative effect between the two drugs and is proposed by the EU note on guidance as a primary efficacy endpoint of choice. The definition of the responder status is different throughout the studies. However, in most cases, the change in pain score that defines a clinically important difference for the individual patient is the most appropriate cut-off value $[20,21]$. Hence, we defined the equivalence margin $(13 \mathrm{~mm})$ of sore throat pain intensity difference at 1 hour, 2 hours and 3 hours after the initial dose as a cut-off point between the responder and nonresponder status. In this respect, the responders maintain sustainable pain relief over the key points of the dosing interval.

As for the safety part, the number of drug-related adverse events was too low to justify a reasonable comparable analysis of the events. Low incidence was expected since both compounds are relatively safe, do not have a systemic effect in the recommended doses and have many years of safe use in their life cycle history. Furthermore, the incidence of individual drug-related adverse events was in majority of cases under $1 \%$ of the study population. Mostly, there were solitary cases of mild intensity. All of them abated by the end of the treatment. Lack of any severe or serious adverse events further corroborates the safety of both formulations and advocate their broad use in the supportive upper respiratory tract infections treatment setting. 
Marko Boh (2021) A Therapeutic Equivalence of Two Formulations of the Fixed Combination of Benzydamine Hydrochloride and Cetylpyridinium Chloride in the Treatment of Sore Throat Associated with Upper Respiratory Tract Infection

In conclusion, this clinical trial has demonstrated that $\mathrm{CPC} / \mathrm{BH}$ lozenges are therapeutically equivalent to $\mathrm{CPC} / \mathrm{BH}$ orosoluble tablets in terms of sore throat treatment. The superior effect of both drugs persisted and was significantly better than placebo over the entire recommended dosing interval. These results were corroborated with the assessment of the percent of subjects who adequately responded to the therapy as well as with disease resolution after 4 and 7 days of treatment. Both drugs turned out to be relatively safe with the low incidence of adverse reactions and lack of severe or serious events.

\section{References}

1. Bisino AL (2011) Acute pharyngitis. N Engl J Med 344: 205-211 [crossref]

2. Hannaford PC, Simpson JA, Bisset AF, et al. (2005) The prevalence of ear, nose and throat problems in the community: results from a national cross-sectional postal survey in Scotland. Fam Pract 22: 227-233 [crossref]

3. Addey D, Shephard A (2012) Incidence, causes, severity and treatment of throat discomfort: a four-region online questionnaire survey. BMC Ear Nose Throat Disord 12: 9 [crossref]

4. Little PS, Wiliamson I, Warner G, et al. (1997) Open randomised trial of prescribing strategies in managing sore throat. BMJ 314: 722-727 [crossref]

5. Butler CC, Rollnick S, Kinnersley P, et al. (1998) Reducing antibiotics for respiratory tract symptoms in primary care: consolidating 》why $\ll$ and considering $\gg$ how $\ll$. $\mathrm{Br}$ J Gen Pract 48: 1865-1870 [crossref]

6. Merianos JJ (1991) Quaternary ammonium antimicrobial compounds. In: Block SS, ed. Disinfection, sterilisation and preservation. 4th ed. Philadelphia, London: Lea \& Febiger p. 225-255

7. Pitten FA, Kramer A (2001) Efficacy of cetylpyridinium chloride used as oropharyngeal antiseptic. Arzneimittelforschung 51: 588-595 [crossref]

8. Quane PA, Graham GG, Ziegler JB (1998) Pharmacology of benzydamine. Inflammopharmacology. 6: 95-107 [crossref]
9. Sardella A, Uglietti D, DemarosI F et al. (1999) Benzydamine hydrochloride oral rinses in management of burning mouth syndrome: A clinical trial. Oral Surg Oral Med Oral Pathol Oral Radiol Endod 88: 683-686 [crossref]

10. Wethington JF (1985) Double-blind study of benzydamine hydrochloride, a new treatment for sore throat. ClinTher 7: 641-646 [crossref]

11. Schachtel BP et al. (1984) Rating scales for analgesics in sore throat. Clin Pharmacol Ther 36: 151-156 [crossref]

12. Schachtel BP et al. (2002) Demonstration of dose response of flurbiprofen lozenges with the sore throat pain model. Clin Pharmacol Ther 71:375-80 [crossref]

13. Schachtel BP, Fillingim JM, Beiter DJ, Lane AC, Schwartz LA (1984) Subjective and objective features of sore throat. Arch Intern Med 144: 497-500 [crossref]

14. Todd KH. Et al. (1996) Clinical significance of reported changes in pain severity. Ann Emerg Med 27: 485-489 [crossref]

15. Herrera D, Santos S, Ferrús J et al. Efficacy of a $0.15 \%$ benzydamine hydrochloride and $0.05 \%$ cetylpyridinium chloride mouth rinse on 4 -day de novo plaque formation. J Clin Peridonto 32; 595-603 [crossref]

16. CPMP/ICH/363/96. Note on guidance on statistical principles for clinical trials. Available at: https://www.ema.europa .eu/en/documents/scientific-guideline/ich-e9-statistical-principles-clinical-trials-step-5_en.pdf, (accessed March 8, 2021).

17. Schachtel BP et al. (1988) Sore throat pain in the evaluation of mild analgesics. Clin Pharmacol Ther 44: 704-711 [crossref]

18. Stahmer SA, Shofer FS, Marino A, Shepherd S, Abbuhl S (1998) Do quantitative changes in pain intensity correlate with pain relief and satisfaction. Acad Emerg Med. 5: 851-857

19. Schachtel BP, Fillingim JM, Lane AC, Thoden WR, Baybutt RI (1991) Caffeine as an analgesic adjuvant. A double-blind study comparing aspirin with caffeine to aspirin and placebo in patients with sore throat. Arch Intern Med 151: 733-737 [crossref]

20. Schachtel BP et al. (2007) Utility and Sensitivity of the Sore Throat Pain Model: Results of a Randomized Controlled Trial on the COX-2 Selective Inhibitor Valdecoxib. Journal of Clinical Pharmacology 47: 860-870 [crossref]

21. Farrar JT, Berlin JA, Strom BL (2003) Clinically important changes in pain outcome measures: a validation study. J Pain Symptom Manag 25: 406-411 [crossref] 